\title{
NORMALIZED EIGENVECTORS OF A PERTURBED LINEAR OPERATOR VIA GENERAL BIFURCATION
}

\author{
RAFFAELE CHIAPPINELLI \\ Dipartimento di Scienze Matematiche ed Informatiche, Pian dei Mantellini 44, I-53100 Siena, \\ Italy - E-mail address: chiappinelli@unisi.it \\ MASSIMO FURI \\ Dipartimento di Matematica Applicata 'G. Sansone', Via S. Marta 3, I-50139 Florence, \\ Italy-E-mail address: massimo.furi@unifi.it \\ and MARIA PATRIZIA PERA \\ Dipartimento di Matematica Applicata 'G. Sansone', Via S. Marta 3, \\ I-50139 Florence, Italy-E-mail address: mpatrizia.pera@unifi.it
}

(Received 29 January, 2007; revised 10 October, 2007; accepted 14 October, 2007)

\begin{abstract}
Let $X$ be a real Banach space, $A: X \rightarrow X$ a bounded linear operator, and $B: X \rightarrow X$ a (possibly nonlinear) continuous operator. Assume that $\lambda=0$ is an eigenvalue of $A$ and consider the family of perturbed operators $A+\varepsilon B$, where $\varepsilon$ is a real parameter. Denote by $S$ the unit sphere of $X$ and let $S_{A}=S \cap \operatorname{Ker} A$ be the set of unit 0 -eigenvectors of $A$. We say that a vector $x_{0} \in S_{A}$ is a bifurcation point for the unit eigenvectors of $A+\varepsilon B$ if any neighborhood of $\left(0,0, x_{0}\right) \in \mathbb{R} \times \mathbb{R} \times X$ contains a triple $(\varepsilon, \lambda, x)$ with $\varepsilon \neq 0$ and $x$ a unit $\lambda$-eigenvector of $A+\varepsilon B$, i.e. $x \in S$ and $(A+\varepsilon B) x=\lambda x$.

We give necessary as well as sufficient conditions for a unit 0 -eigenvector of $A$ to be a bifurcation point for the unit eigenvectors of $A+\varepsilon B$. These conditions turn out to be particularly meaningful when the perturbing operator $B$ is linear. Moreover, since our sufficient condition is trivially satisfied when $\operatorname{Ker} A$ is one-dimensional, we extend a result of the first author, under the additional assumption that $B$ is of class $C^{2}$.
\end{abstract}

2000 Mathematics Subject Classification.

1. Introduction. The intent of this paper is a nontrivial extension, under some additional regularity assumptions, of a perturbation result on nonlinear spectral theory due to the first author (see [1, Theorem 2]). This result can be (re)formulated as follows.

THEOREM 1.1. Let $T$ be a selfadjoint bounded operator on a real Hilbert space $H$, and $B: H \rightarrow H$ a Lipschitz continuous operator. Let $S$ denote the unit sphere of $H$ and assume that $\lambda_{0}$ is an isolated simple eigenvalue of $T$. Then, given one of the two unit $\lambda_{0}$-eigenvectors of $H$, say $x_{0}$, there exist $\sigma>0, \delta>0$, and a neighborhood $U$ of $x_{0}$ such that for every $\varepsilon \in(-\sigma, \sigma)$ one can find a unique $\lambda_{\varepsilon} \in\left(\lambda_{0}-\delta, \lambda_{0}+\delta\right)$ and a unique $x_{\varepsilon} \in S \cap U$ such that

$$
(T+\varepsilon B) x_{\varepsilon}=\lambda_{\varepsilon} x_{\varepsilon}
$$

Moreover, the maps $\varepsilon \mapsto \lambda_{\varepsilon}$ and $\varepsilon \mapsto x_{\varepsilon}$ are Lipschitz continuous. 
In some sense, this result asserts that an isolated unit eigenvector of $T$ cannot be destroyed by a small perturbation $\varepsilon B$, but just displaced from its original position, still remaining on the unit sphere $S$ of $H$.

It is natural to ask what happens if one drops the hypothesis that the eigenvalue $\lambda_{0}$ is simple. Our feeling is that if its multiplicity is odd (algebraic and geometric multiplicities are the same in the selfadjoint case), then at least one unit eigenvector of $T+\varepsilon B$ survives, provided that the coefficient $\varepsilon$ is sufficiently small. To be more precise, our conjecture is that in this case there exists a sequence $\left\{\left(\varepsilon_{i}, \lambda_{i}, x_{i}\right)\right\}$ in $\mathbb{R} \times \mathbb{R} \times S$ which converges to a point $\left(0, \lambda_{0}, x_{0}\right)$ and such that

$$
\left(T+\varepsilon_{i} B\right) x_{i}=\lambda_{i} x_{i}, \quad \varepsilon_{i} \neq 0, \quad \forall i \in \mathbb{N} .
$$

Clearly, if this happens, $x_{0}$ must be a unit eigenvector of $T$ with eigenvalue $\lambda_{0}$.

Unfortunately, we are not able, so far, to prove or disprove this conjecture which, we believe, is related to the fact that the Euler-Poincare characteristic of the even dimensional sphere $S \cap \operatorname{Ker}\left(T-\lambda_{0} I\right)$ is nonzero ( $I$ denotes the identity on $H$ ).

In this work, we tackle a different, but related, problem in the general case in which the multiplicity of the isolated eigenvalue $\lambda_{0}$ is finite. Then, the kernel of the operator $A=T-\lambda_{0} I$ is nontrivial (i.e., $0 \in \mathbb{R}$ is an eigenvalue of $A$ ) and finite dimensional. Moreover, the unperturbed operator $A$ is Fredholm of index zero (in fact, its image is the orthogonal complement to its kernel). We consider the set $\Sigma \subseteq \mathbb{R} \times \mathbb{R} \times H$ of the solutions $(\varepsilon, \lambda, x)$ of the problem

$$
(A+\varepsilon B) x=\lambda x, \quad x \in S,
$$

and we regard the distinguished subset $M=\{(0,0, x) \in \Sigma: A x=0\}$ of $\Sigma$ as the set of trivial solutions of (1.1). Since $(\Sigma, M)$ is a topological pair (i.e. $M$ is a subspace of the topological space $\Sigma$ ), according to [3], an element $p_{0}=\left(0,0, x_{0}\right) \in M$ is called a bifurcation point of this pair (or, equivalently, of problem (1.1)) if any neighborhood of $p_{0}$ contains an element of $\Sigma \backslash M$, which, in our case, is a nontrivial solution of (1.1). Since the finite dimensional sphere $S_{A}=S \cap \operatorname{Ker} A$ (of the normalized 0-eigenvectors of $A$ ) may be identified with the distinguished set $M$, for the sake of simplicity we will say that an element $x_{0} \in S_{A}$ is a bifurcation point of (1.1) if so is $p_{0}=\left(0,0, x_{0}\right)$. From two results in [3] about this general point of view in bifurcation theory we will deduce necessary as well as sufficient conditions for a normalized 0 -eigenvector of $A$ to be a bifurcation point of (1.1). Since these conditions are trivially satisfied when the sphere $S_{A}$ is zero-dimensional (that is, when 0 is a simple eigenvalue of $A$ ), we extend Theorem 1.1 of the first author, under the additional assumption that $B$ is of class $C^{2}$, which is required in order to apply a sufficient condition for bifurcation given in [3].

Our results are particularly meaningful when the perturbing operator $B$ is linear. In this case a necessary condition for $x_{0} \in S_{A}$ to be a bifurcation point is that $x_{0}$ is an eigenvector (associated with a real eigenvalue) for the finite dimensional operator

$$
\hat{B}: \operatorname{Ker} A \rightarrow \operatorname{Ker} A
$$

defined by $x \mapsto \pi(B x)$, where $\pi$ is the orthogonal projection onto $\operatorname{Ker} A$.

Incidentally, we observe that, in the case when $\operatorname{Ker} A$ is odd dimensional, this necessary condition does not contradict our conjecture about the existence of a bifurcation point: in this case, the characteristic polynomial of the endomorphism $\hat{B}$ has a real root. The same necessary condition shows that when the dimension of 
Ker $A$ is even, one can always find a bounded linear operator $B$ such that (1.1) does not have bifurcation points: it is sufficient to define $B$ with $\hat{B}$ without real eigenvalues.

Still in the case of $B$ linear, a sufficient condition for $x_{0} \in S_{A}$ to be a bifurcation point is that $x_{0}$ is an eigenvector of $\hat{B}$ corresponding to a simple (real) eigenvalue.

For the sake of generality, as well as simplicity in some applications, we will deal with operators between Banach spaces instead of confining ourselves to the context of Hilbert spaces. Thus, we shall consider (1.1) where $A: X \rightarrow X(X$ a Banach space) is a Fredholm operator of index zero with nontrivial kernel and such that $\operatorname{Ker} A \oplus \operatorname{Im} A=$ $X$; in fact, we will consider a more general situation, see (3.1) below.

In detail, the plan of this paper is as follows.

In Section 2 we recall the notion of nonlinear Fredholm map between Banach spaces, a concept which is required in order to state precisely, as Theorem 2.1 and Theorem 2.2, the two bifurcation results from [3] mentioned above. These results regard, respectively, a necessary condition and a sufficient condition for bifurcation of a pair $\left(f^{-1}(0), M\right)$, where $f$ is a Fredholm map between Banach spaces and $M \subseteq f^{-1}(0)$ is a differentiable manifold (Theorem 2.2 extends the well-known Crandall-Rabinowitz sufficient conditions for bifurcation given in [2]).

Section 3 is devoted to statement and proof of our main results: Theorem 3.2 and Theorem 3.4. Essentially these are, respectively, the versions of Theorem 2.1 and Theorem 2.2 which are appropriate to deal with the operator equation (1.1) in order to obtain necessary as well as sufficient conditions for bifurcation of unit vectors for the above equation.

Section 4 contains some useful reformulations of our main results, whose statements, although expressed in Section 3 in a meaningful canonical form, are unfriendly for applications. These new formulations involve the choice of linear coordinates for $\operatorname{Ker} A$ and $\operatorname{coKer} A$.

Finally, in Section 5, we discuss the existence of nontrivial $2 \pi$-periodic solutions of the differential equation

$$
x^{\prime \prime}+x+\varepsilon\left(t x+x^{2}\right)=\lambda x,
$$

which we see as a simple, yet nontrivial, example to test the conclusions of our theory. Here the eigenspace of the unperturbed linear operator is two-dimensional.

2. Notation and preliminaries. Let $E$ and $F$ be two real Banach spaces and let $U$ be an open subset of $E$. Given a $C^{1}$ map $f: U \rightarrow F$, the (first) derivative of $f$ at $u \in U$ will be denoted by $f^{\prime}(u)$. When $E=E_{1} \times E_{2}$, the partial derivative with respect to the first (respectively, the second) variable at $\left(u_{1}, u_{2}\right)$ will be indicated with $\partial_{1} f\left(u_{1}, u_{2}\right)$ (respectively, $\partial_{2} f\left(u_{1}, u_{2}\right)$ ). Because of the linearity of the (total) derivative $f^{\prime}\left(u_{1}, u_{2}\right)$, for any pair of vectors $\left(\dot{u}_{1}, \dot{u}_{2}\right) \in E_{1} \times E_{2}$, one has

$$
f^{\prime}\left(u_{1}, u_{2}\right)\left(\dot{u}_{1}, \dot{u}_{2}\right)=\partial_{1} f\left(u_{1}, u_{2}\right) \dot{u}_{1}+\partial_{2} f\left(u_{1}, u_{2}\right) \dot{u}_{2} .
$$

In particular, if $E_{1}=\mathbb{R}$, the partial derivative $\partial_{1} f\left(u_{1}, u_{2}\right)$, which is actually a linear operator from $\mathbb{R}$ to $F$, will be identified with the vector $\partial_{1} f\left(u_{1}, u_{2}\right)(1) \in F$. With this notation, for $f^{\prime}\left(u_{1}, u_{2}\right)$ one has the equality

$$
f^{\prime}\left(u_{1}, u_{2}\right)\left(\dot{u}_{1}, \dot{u}_{2}\right)=\dot{u}_{1} \partial_{1} f\left(u_{1}, u_{2}\right)+\partial_{2} f\left(u_{1}, u_{2}\right) \dot{u}_{2},
$$

where $\left(\dot{u}_{1}, \dot{u}_{2}\right) \in \mathbb{R} \times E_{2}$. 
The second derivative of a $C^{2} \operatorname{map} f: U \subseteq E \rightarrow F$ at $p \in U$ is a symmetric bilinear operator from $E$ to $F$, i.e. an element of the Banach space $L_{s}^{2}(E, F)$, and will be denoted by $f^{\prime \prime}(p)$. A practical method for its computation is the following: given $\dot{u}, \dot{v} \in E$, consider the function of two real variables $\sigma(r, s)=f(p+r \dot{u}+s \dot{v})$; then,

$$
f^{\prime \prime}(p)(\dot{u}, \dot{v})=\frac{\partial^{2} \sigma}{\partial r \partial s}(0,0) .
$$

Let $L(E, F)$ be the Banach space of the bounded linear operators from $E$ into $F$. We recall that an element $L \in L(E, F)$ is called Fredholm if both $\operatorname{Ker} L$ and $\operatorname{coKer} L:=$ $F / \operatorname{Im} L$ have finite dimensions (consequently, $\operatorname{Im} L$ must be closed). The index of $L$ is the integer

$$
\text { ind } L=\operatorname{dim} \operatorname{Ker} L-\operatorname{dim} \operatorname{coKer} L \text {. }
$$

The following properties of Fredholm operators are well-known and will be used in the sequel:

i) the set of Fredholm operators from $E$ into $F$ of a given index is open in $L(E, F)$;

ii) if $L_{1}: E \rightarrow F$ and $L_{2}: F \rightarrow G$ are Fredholm operators, then the composition $L_{2} L_{1}$ is Fredholm and ind $L_{2} L_{1}=$ ind $L_{1}+$ ind $L_{2}$;

iii) if $L: E \rightarrow F$ is Fredholm and $H: E \rightarrow F$ is a compact linear operator, then $L+H$ is Fredholm and ind $(L+H)=$ ind $L$.

We recall that a (nonlinear) map $f: U \subseteq E \rightarrow F$ is said to be Fredholm of index $n \in \mathbb{Z}$ if it is of class $C^{1}$ and $f^{\prime}(u)$ is Fredholm of index $n$ for all $u \in U$; it is simply called Fredholm if it is Fredholm of index $n$ for some $n$.

If $f: U \rightarrow F$ is $C^{1}$, a point $v \in F$ is a regular value of $f$ if $f^{\prime}(u)$ is surjective for all $u \in f^{-1}(v)$. If $f$ is Fredholm of class $C^{k}$ and $v$ is a regular value of $f$, then the Implicit Function Theorem implies that $f^{-1}(v)$ is a $C^{k}$-submanifold of $E$ with $\operatorname{dim} f^{-1}(v)=$ ind $f$ (see e.g. [6], [7]). Moreover, given $u \in f^{-1}(v)$, the surjectivity of $f^{\prime}(u)$ implies that the tangent space $T_{u} f^{-1}(v)$ of $f^{-1}(v)$ at $u$ coincides with $\operatorname{Ker} f^{\prime}(u)$.

Let $f: U \rightarrow F$ be of class $C^{1}$. Given a differentiable manifold $M \subseteq f^{-1}(0)$, regard $M$ as the set of trivial solutions of the equation $f(u)=0$, so that $f^{-1}(0) \backslash M$ represents the set of nontrivial solutions. An element $p \in M$ is a bifurcation point (from $M$ ) of $f(u)=0$ (or, equivalently, of the topological pair $\left(f^{-1}(0), M\right)$ ) if any neighborhood of $p$ contains elements of $f^{-1}(0) \backslash M$.

In what follows, we will make use of two bifurcation results (Theorems 2.1 and 2.2 below) obtained in [3]. To understand the meaning of these results, observe that the condition $M \subseteq f^{-1}(0)$ implies that, for any $u \in M$, the tangent space $T_{u} M$ of $M$ at $u$ is contained in $\operatorname{Ker} f^{\prime}(u)$.

The following result provides a meaningful necessary condition for an element $p \in M$ to be a bifurcation point of the topological pair $\left(f^{-1}(0), M\right)$. The map $f$ is assumed to be Fredholm (of any given index), and no relation between ind $f$ and $\operatorname{dim} M$ is assumed.

THEOREM 2.1. Let $f: U \subseteq E \rightarrow F$ be a $C^{1}$ Fredholm map defined on an open subset $U$ of a Banach space $E$ into a Banach space $F$ and let $M$ be a $C^{1}$ manifold contained in $f^{-1}(0)$. If $p \in M$ is a bifurcation point (from $M$ ) for the equation $f(u)=0$, then $T_{p} M$ is a proper subspace of $\operatorname{Ker} f^{\prime}(p)$.

The following sufficient condition for bifurcation should not be regarded as a result in the so-called "several-parameter bifurcation" (see e.g. [9]) since the dimension 
of the manifold $M$ of the trivial solutions of the equation $f(x)=0$ does not necessarily coincide with the index of the Fredholm map $f$ (which below is assumed to be one). It should be regarded, instead, as an extension of the Crandall-Rabinowitz Bifurcation Theorem, in which $\operatorname{dim} M=1$ (see [2]), as well as an extension of a result obtained independently in [8] and in [4], in which $M$ is a finite dimensional subspace of the Banach space $E$.

TheOREM 2.2. Let $f: U \subseteq E \rightarrow F$ be a $C^{2}$ Fredholm map of index one defined on an open subset $U$ of a Banach space $E$ into a Banach space $F$. Let $M$ be a $C^{2}$ manifold contained in $f^{-1}(0)$ and let $p \in M$ be such that $\operatorname{dim} \operatorname{Ker} f^{\prime}(p)=\operatorname{dim} T_{p} M+1$. Choose any $\dot{u} \in \operatorname{Ker} f^{\prime}(p) \backslash T_{p} M$. Then $p$ is a bifurcation point (from $M$ ) for the equation $f(u)=0$, provided that

$$
\dot{v} \in T_{p} M \quad \text { and } \quad f^{\prime \prime}(p)(\dot{u}, \dot{v}) \in \operatorname{Im} f^{\prime}(p) \Longrightarrow \dot{v}=0 .
$$

REMARK 2.3. In [5] it was proved that the condition (2.1) does not depend on the choice of the vector $\dot{u} \in \operatorname{Ker} f^{\prime}(p) \backslash T_{p} M$.

Notice that if in Theorem 2.2 the manifold $M$ is assumed to be a singleton $\{p\}$, then condition (2.1) is automatically satisfied, since $T_{p} M=\{0\}$. However, in this case, $\operatorname{dim} \operatorname{Ker} f^{\prime}(p)=1$ and, consequently, $f^{\prime}(p)$ is a surjective operator. Thus, the assertion could be directly deduced from the Implicit Function Theorem, which implies that $f^{-1}(0)$, in a neighborhood of $p$, is a 1-dimensional manifold.

REMARK 2.4. An equivalent formulation of the condition (2.1) is the following:

$$
\dot{v} \in T_{p} M \quad \text { and } \quad \pi f^{\prime \prime}(p)(\dot{u}, \dot{v})=0 \Longrightarrow \dot{v}=0,
$$

where $\pi: F \rightarrow F / \operatorname{Im} f^{\prime}(p)$ denotes the canonical projection. Observe also that any projection parallel to $\operatorname{Im} f^{\prime}(p)$ onto a direct summand of $\operatorname{Im} f^{\prime}(p)$, or any bounded linear operator $Q: F \rightarrow \mathbb{R}^{m}\left(m=\operatorname{dim} T_{p} M\right)$ such that $\operatorname{Ker} Q=\operatorname{Im} f^{\prime}(p)$, would play the same role as $\pi$.

3. Main results. Let $X$ and $Y$ be two real Banach spaces and consider the system

$$
\left\{\begin{array}{l}
A x+\varepsilon B(x)=\lambda C x \\
g(x)=0
\end{array}\right.
$$

where $A: X \rightarrow Y$ and $C: X \rightarrow Y$ are bounded linear operators, $\varepsilon$ and $\lambda$ are real parameters, $B: X \rightarrow Y$ and $g: X \rightarrow \mathbb{R}$ are continuous maps. We assume that $A$ is Fredholm of index zero with nontrivial kernel and that

$$
C(\operatorname{Ker} A) \oplus \operatorname{Im} A=Y .
$$

As a consequence, $A-\lambda C$ is onto for $\lambda \neq 0$ small and, thus, one-to-one, since it is Fredholm of index zero (being close to $A$ ). This shows that $\lambda=0$ is an isolated eigenvalue for the problem $A x=\lambda C x$. Moreover, as is easy to verify, $\operatorname{dim} C(\operatorname{Ker} A)=$ $\operatorname{codim} \operatorname{Im} A=\operatorname{dim} \operatorname{Ker} A$. Thus, assumption (3.2) is also equivalent to $C(\operatorname{Ker} A)+$ $\operatorname{Im} A=Y$.

A solution of (3.1) is a triple $u=(\varepsilon, \lambda, x)$ in the Banach space $E=\mathbb{R} \times \mathbb{R} \times X$ satisfying (3.1). Clearly, for $\varepsilon=\lambda=0$, the triple $(0,0, x)$ is a solution if and only if $x$ 
belongs to the set

$$
S_{A}:=\operatorname{Ker} A \cap g^{-1}(0)
$$

Assume that $g^{-1}(0)$ surrounds the origin in the following sense: for all $x \neq 0$, there exists $s>0$ such that $g(s x)=0$. Also assume that $g$ is of class $C^{k}(k \geq 1)$ on an open neighborhood of $g^{-1}(0)$ and $g^{\prime}(x) x \neq 0$ for any $x \in g^{-1}(0)$. This last condition ensures that 0 is a regular value both for $g$ and for the restriction of $g$ to $\operatorname{Ker} A$ (observe that the functional $g^{\prime}(x)$ is nonzero for any $x \in g^{-1}(0)$ and is nonzero also its restriction to Ker $A$ for any $x \in S_{A}$ ). Consequently, $g^{-1}(0)$ is a $C^{k}$ manifold of codimension 1 in $X$ and $S_{A}$ is a nonempty $C^{k}$ manifold of dimension

$$
\operatorname{dim} S_{A}=\operatorname{dim} \operatorname{Ker} A-1 .
$$

A significant and sufficiently general example of function $g$ is $g(x)=|x|^{2}-1$, where $|\cdot|$ is a smooth norm of the space $X$, but not necessarily the Banach norm of $X$, as in the example of Section 5. The convenience of considering norms which are not necessarily complete is due to the fact that the Banach norm of the space $X$ may not be differentiable.

Any solution of (3.1) that belongs to the manifold

$$
M:=\{0\} \times\{0\} \times S_{A}
$$

will be called trivial. Thus, any solution $(\varepsilon, \lambda, x)$ with $(\varepsilon, \lambda) \neq(0,0)$ will be a nontrivial solution. Identifying $S_{A}$ with $M$, an eigenvector $\bar{x} \in S_{A}$ of the operator $A$ (corresponding to the eigenvalue $\lambda=0$ ) will be called a bifurcation point (from $\left.S_{A}\right)$ of system (3.1) if any neighborhood of $p=(0,0, \bar{x})$ in $E=\mathbb{R} \times \mathbb{R} \times X$ contains a nontrivial solution. In this case we will also say that $p=(0,0, \bar{x})$ is a bifurcation point from $M$. Since, as already remarked, $A-\lambda C$ is invertible for $\lambda \neq 0$ small, say $0<|\lambda|<\lambda_{0}$, one gets that system (3.1) has no solutions for $\varepsilon=0$ and $0<|\lambda|<\lambda_{0}$. Thus, any nontrivial solution $(\varepsilon, \lambda, x)$ close to a bifurcation point $p=(0,0, \bar{x})$ must have $\varepsilon \neq 0$.

We are interested in obtaining necessary as well as sufficient conditions for a 0 eigenvector of $A$ to be a bifurcation point of (3.1). To this end, let us interpret system (3.1) above in the abstract setting of Theorem 2.1 by considering the Banach spaces $E=\mathbb{R} \times \mathbb{R} \times X$ and $F=Y \times \mathbb{R}$ and by defining $f: E \rightarrow F$ as

$$
f(\varepsilon, \lambda, x)=(A x+\varepsilon B(x)-\lambda C x, g(x)) .
$$

The manifold $M=\{0\} \times\{0\} \times S_{A}$, which is clearly a subset of $f^{-1}(0)$, will be regarded as the set of trivial solutions of (3.1) or, equivalently, of the equation

$$
f(\varepsilon, \lambda, x)=0 .
$$

By using the terminology introduced above, an eigenvector $\bar{x} \in S_{A}$ is a bifurcation point (from $S_{A}$ ) of system (3.1) if and only if $p=(0,0, \bar{x}) \in M$ is a bifurcation point (from $M$ ) of equation (3.3). Moreover, $\operatorname{dim} M=\operatorname{dim} S_{A}=\operatorname{dim} \operatorname{Ker} A-1$.

Lemma 3.1 below shows that $f$ is a Fredholm map.

LEMMA 3.1. Assume B and $g$ of class $C^{k}(k \geq 1)$ on an open neighborhood of $g^{-1}(0)$. Then $f$ is $C^{k}$ and Fredholm of index one on an open neighborhood of $M$. 
Proof. Since $B$ and $g$ are $C^{k}$ on an open neighborhood of $g^{-1}(0)$ in $X$, then $f$ is $C^{k}$ in a suitable open neighborhood of $M \subseteq f^{-1}(0)$ in $E=\mathbb{R} \times \mathbb{R} \times X$.

The map $f$ can be seen as the sum of the linear map $L(\varepsilon, \lambda, x)=(A x, 0)$ with the map $h(\varepsilon, \lambda, x)=(\varepsilon B(x)-\lambda C x, g(x))$. Since $A$ is Fredholm of index 0 , then $L$ is clearly Fredholm of index 1. Moreover, computing the derivative of $h$ at a point $(0,0, x)$ for which $g(x)=0$, one gets

$$
h^{\prime}(0,0, x)(\dot{\varepsilon}, \dot{\lambda}, \dot{x})=\left(\dot{\varepsilon} B(x)-\dot{\lambda} C x, g^{\prime}(x) \dot{x}\right) .
$$

Hence, $h^{\prime}(0,0, x)$ has finite dimensional image. Therefore, as already observed in the preliminaries, $f=L+h$ is Fredholm of index 1 at any point of $\{0\} \times\{0\} \times g^{-1}(0)$ and, being $C^{k}$, is still of index 1 on an open neighborhood of $M$, as claimed.

Theorem 3.2 below provides a necessary condition for bifurcation in which a canonical (i.e., independent of the coordinate systems) finite dimensional reduction between $\operatorname{Ker} A$ and $\operatorname{coKer} A$ is done.

TheOREM 3.2. Assume $B$ of class $C^{1}$ on an open neighborhood of $g^{-1}(0)$. If $\bar{x} \in S_{A}$ is a bifurcation point of (3.1), then there exists $\bar{\mu} \in \mathbb{R}$ such that

$$
B(\bar{x})-\bar{\mu} C \bar{x} \in \operatorname{Im} A .
$$

Proof. As above, let $f: \mathbb{R} \times \mathbb{R} \times X \rightarrow Y \times \mathbb{R}$ be the map

$$
f(\varepsilon, \lambda, x)=(A x+\varepsilon B(x)-\lambda C x, g(x))
$$

and let $M \subseteq f^{-1}(0)$ be the set

$$
M=\{0\} \times\{0\} \times S_{A} .
$$

As stated in Lemma 3.1, $f$ is $C^{1}$ and Fredholm of index 1 on an open neighborhood of the $C^{1}$ manifold $M$. Our aim is to apply Theorem 2.1 to $f$ and $M$. To this end, let $X_{1}$ and $Y_{2}$ be closed subspaces of $X$ and $Y$, respectively, such that $X=X_{1} \oplus \operatorname{Ker} A$ and $Y=\operatorname{Im} A \oplus Y_{2}$. Since the space $X_{1}$ is Banach, $X$ can be identified with $X_{1} \times$ $\operatorname{Ker} A$ via the operator $\left(x_{1}, x_{2}\right) \mapsto x_{1}+x_{2}$, whose inverse is continuous because of the Inverse Function Theorem. Analogously, $Y$ will be identified with $\operatorname{Im} A \times Y_{2}$. In this decomposition, we have $x=\left(x_{1}, x_{2}\right)$ and system (3.1) can be written in block-matrix form as follows:

$$
\left\{\begin{array}{l}
\left(\begin{array}{ll}
A_{11} & 0 \\
0 & 0
\end{array}\right)\left(\begin{array}{l}
x_{1} \\
x_{2}
\end{array}\right)+\varepsilon\left(\begin{array}{l}
B_{1}\left(x_{1}, x_{2}\right) \\
B_{2}\left(x_{1}, x_{2}\right)
\end{array}\right)-\lambda\left(\begin{array}{l}
C_{1}\left(x_{1}, x_{2}\right) \\
C_{2}\left(x_{1}, x_{2}\right)
\end{array}\right)=\left(\begin{array}{l}
0 \\
0
\end{array}\right) \\
g\left(x_{1}, x_{2}\right)=0
\end{array}\right.
$$

Also observe that, since $A$ is Fredholm of index zero, then $\operatorname{dim} Y_{2}=\operatorname{dim} \operatorname{Ker} A$. Let $\bar{x} \in S_{A}$ be the given bifurcation point. Then, since $S_{A} \subseteq \operatorname{Ker} A$, we have $\bar{x}=(0, q)$, for some $q \in \operatorname{Ker} A$. Thus, in the notation of Theorem 2.1 the element $p=(0,0,0, q)$ is a bifurcation point of (3.3) from $M$. Consequently, by Theorem 2.1, $T_{p} M$ is a proper subset of $\operatorname{Ker} f^{\prime}(p)$. Let us compute explicitly $\operatorname{Ker} f^{\prime}(p)$ and $T_{p} M$. It is easy to see that 
a vector $\left(\dot{\varepsilon}, \dot{\lambda}, \dot{x}_{1}, \dot{x}_{2}\right) \in \mathbb{R} \times \mathbb{R} \times X_{1} \times \operatorname{Ker} A$ belongs to $\operatorname{Ker} f^{\prime}(p)$ if and only if

$$
\left\{\begin{array}{l}
\left(\begin{array}{ll}
A_{11} & 0 \\
0 & 0
\end{array}\right)\left(\begin{array}{l}
\dot{x}_{1} \\
\dot{x}_{2}
\end{array}\right)+\dot{\varepsilon}\left(\begin{array}{l}
B_{1}(0, q) \\
B_{2}(0, q)
\end{array}\right)-\dot{\lambda}\left(\begin{array}{l}
C_{1}(0, q) \\
C_{2}(0, q)
\end{array}\right)=\left(\begin{array}{l}
0 \\
0
\end{array}\right) \\
\partial_{1} g(0, q) \dot{x}_{1}+\partial_{2} g(0, q) \dot{x}_{2}=0
\end{array}\right.
$$

or, equivalently, if and only if

$$
\left\{\begin{array}{l}
A_{11} \dot{x}_{1}+\dot{\varepsilon} B_{1}(0, q)-\dot{\lambda} C_{1}(0, q)=0 \\
\dot{\varepsilon} B_{2}(0, q)=\dot{\lambda} C_{2}(0, q) \\
\partial_{1} g(0, q) \dot{x}_{1}+\partial_{2} g(0, q) \dot{x}_{2}=0 .
\end{array}\right.
$$

Moreover, the tangent space of $M$ at $p$ is given by

$$
T_{p} M=\left\{\left(0,0,0, \dot{x}_{2}\right): \partial_{2} g(0, q) \dot{x}_{2}=0\right\} .
$$

As already observed, since $p$ is a bifurcation point of (3.3) from $M$, by Theorem 2.1, $\operatorname{Ker} f^{\prime}(p)$ contains properly $T_{p} M$. Thus, there exists $\left(\dot{\varepsilon}, \dot{\lambda}, \dot{x}_{1}, \dot{x}_{2}\right) \in \operatorname{Ker} f^{\prime}(p) \backslash T_{p} M$. Let us show that $\dot{\varepsilon} \neq 0$. Suppose that $\dot{\varepsilon}=0$. Since $\bar{x} \neq 0$, condition (3.2) clearly implies $C \bar{x} \notin \operatorname{Im} A$. Hence, $C_{2}(0, q) \neq 0$ and, thus, $\dot{\lambda}=0$. Therefore, from (3.5), one gets $A_{11} \dot{x}_{1}=0$ and, thus, $\dot{x}_{1}=0$ since $A_{11}$ is an isomorphism between $X_{1}$ and $\operatorname{Im} A$. Consequently, it turns out that $\left(\dot{\varepsilon}, \dot{\lambda}, \dot{x}_{1}, \dot{x}_{2}\right)$ is of the form $\left(0,0,0, \dot{x}_{2}\right)$, i.e. an element of $T_{p} M$. A contradiction. This proves that $\dot{\varepsilon}$ must be different from 0 . Hence, by dividing by $\dot{\varepsilon}$ the second equation in (3.6) and by setting $\bar{\mu}=\dot{\lambda} / \dot{\varepsilon}$, one gets

$$
B_{2}(0, q)-\bar{\mu} C_{2}(0, q)=0,
$$

which is equivalent to

$$
B(\bar{x})-\bar{\mu} C \bar{x} \in \operatorname{Im} A,
$$

as claimed.

REMARK 3.3. By introducing the canonical projection $\pi: Y \rightarrow \operatorname{coKer} A$, the necessary condition of Theorem 3.2 above can be expressed equivalently as follows: there exists $\bar{\mu} \in \mathbb{R}$ such that

$$
\pi B(\bar{x})=\bar{\mu} \pi C \bar{x} .
$$

Theorem 3.4 below provides a sufficient condition for $\bar{x} \in S_{A}$ to be a bifurcation point of (3.1). As for the necessary condition given in Theorem 3.2, this result represents the canonical version of the sufficient condition, in the sense that it is stated without any choice of coordinate systems on $\operatorname{Ker} A$ and $\operatorname{coKer} A$.

THEOREM 3.4. Assume $B$ and $g$ of class $C^{2}$ on an open neighborhood of $g^{-1}(0)$. Let $\bar{x} \in S_{A}$ and $\bar{\mu} \in \mathbb{R}$ be such that $B(\bar{x})-\bar{\mu} C \bar{x} \in \operatorname{Im} A$. Then $\bar{x}$ is a bifurcation point of (3.1), provided that

$$
A \dot{h}=0, \quad g^{\prime}(\bar{x}) \dot{h}=0 \quad \text { and } \quad B^{\prime}(\bar{x}) \dot{h}-\bar{\mu} C \dot{h} \in \operatorname{span}\{\operatorname{Im} A, C \bar{x}\} \Longrightarrow \dot{h}=0 .
$$

Proof. Let $f$ and $M$ be as in Lemma 3.1 and, as already done in the proof of Theorem 3.2, consider the decompositions $X=X_{1} \times \operatorname{Ker} A$ and $Y=\operatorname{Im} A \times Y_{2}$, with $\bar{x}=(0, q)$ and $p=(0,0,0, q)$. By Lemma $3.1, f$ is a $C^{2}$ Fredholm map of index 1 on 
an open neighborhood of the $C^{2}$ manifold $M$. To get the assertion, our aim is to apply Theorem 2.2. Let us show first that we have

$$
\operatorname{dim} \operatorname{Ker} f^{\prime}(p)=\operatorname{dim} T_{p} M+1
$$

To this end, consider the nonzero functional on $\operatorname{Ker} f^{\prime}(p)$ given by $\left(\dot{\varepsilon}, \dot{\lambda}, \dot{x}_{1}, \dot{x}_{2}\right) \mapsto \dot{\varepsilon}$. Its kernel, which is clearly 1 -codimensional, consists of those solutions $\left(\dot{\varepsilon}, \dot{\lambda}, \dot{x}_{1}, \dot{x}_{2}\right)$ of system (3.6) possessing $\dot{\varepsilon}=0$. The same argument used in the proof of Theorem 3.2 shows that such solutions must be of the form $\left(0,0,0, \dot{x}_{2}\right)$ with $\partial_{2} g(0, q) \dot{x}_{2}=0$, i.e. belong to $T_{p} M$. Hence, $T_{p} M$ coincides with the kernel of a nonzero functional on $\operatorname{Ker} f^{\prime}(p)$ and, thus, is 1-codimensional. This proves (3.7).

Let $\bar{\mu}$ be as in the assumption and take $\left(\dot{\bar{x}}_{1}, \dot{\bar{x}}_{2}\right) \in X_{1} \times \operatorname{Ker} A$ such that the vector $\dot{u}=\left(1, \bar{\mu}, \dot{\bar{x}}_{1}, \dot{\bar{x}}_{2}\right)$ satisfies system (3.6). Clearly, from the first equation on (3.6), it turns out that $\dot{\bar{x}}_{1}$ is unique (recall that $A_{11}$ is an isomorphism). Moreover, the existence of $\dot{\bar{x}}_{2} \in \operatorname{Ker} A$ such that $\partial_{1} g(0, q) \dot{\bar{x}}_{1}+\partial_{2} g(0, q) \dot{\bar{x}}_{2}=0$ is due to the fact that the functional $\dot{x}_{2} \mapsto \partial_{2} g(0, q) \dot{x}_{2}$ is onto since $\partial_{2} g(0, q) \neq 0$. This means that $\dot{u}$ belongs to $\operatorname{Ker} f^{\prime}(p)$ and, since its first component is nonzero, it is not tangent to $M$ at $p$. Thus, as needed for applying Theorem 2.2, we have chosen $\dot{u} \in \operatorname{Ker} f^{\prime}(p) \backslash T_{p} M$.

Let now $\varphi: S_{A} \rightarrow F \times \mathbb{R}$ be given by $\varphi(x)=f^{\prime}(0,0, x) \dot{u}$. Since, in the identification $X=X_{1} \times \operatorname{Ker} A$, any $x \in S_{A}$ is of the form $x=\left(0, x_{2}\right)$, we have

$$
\varphi\left(0, x_{2}\right)=\left(\begin{array}{l}
A_{11} \dot{\bar{x}}_{1}+B_{1}\left(0, x_{2}\right)-\bar{\mu} C_{1}\left(0, x_{2}\right) \\
B_{2}\left(0, x_{2}\right)-\bar{\mu} C_{2}\left(0, x_{2}\right) \\
\partial_{1} g\left(0, x_{2}\right) \dot{\bar{x}}_{1}+\partial_{2} g\left(0, x_{2}\right) \dot{\bar{x}}_{2}
\end{array}\right) .
$$

Let us compute the derivative of $\varphi$ at $(0, q)$ along a vector $\dot{h} \in T_{\bar{x}} S_{A}$. Clearly, $\dot{h} \in T_{\bar{x}} S_{A}$ if and only if $A \dot{h}=0$ and $g^{\prime}(\bar{x}) \dot{h}=0$, i.e. if and only if $\dot{h}=\left(0, \dot{x}_{2}\right)$ with $\partial_{2} g(0, q) \dot{x}_{2}=0$. We get

$$
\varphi^{\prime}(0, q)\left(0, \dot{x}_{2}\right)=\left(\begin{array}{l}
\partial_{2} B_{1}(0, q) \dot{x}_{2}-\bar{\mu} C_{1}\left(0, \dot{x}_{2}\right) \\
\partial_{2} B_{2}(0, q) \dot{x}_{2}-\bar{\mu} C_{2}\left(0, \dot{x}_{2}\right) \\
\psi^{\prime}(0, q) \dot{x}_{2}
\end{array}\right),
$$

where $\psi: S_{A} \rightarrow \mathbb{R}$ is given by

$$
\left(0, x_{2}\right) \mapsto \partial_{1} g\left(0, x_{2}\right) \dot{\bar{x}}_{1}+\partial_{2} g\left(0, x_{2}\right) \dot{\bar{x}}_{2} .
$$

By denoting $\dot{v}=(0,0, \dot{h})=\left(0,0,0, \dot{x}_{2}\right)$, we obviously have

$$
\varphi^{\prime}(0, q) \dot{h}=f^{\prime \prime}(0,0,0, q)(\dot{u},(0,0, \dot{h}))=f^{\prime \prime}(p)(\dot{u}, \dot{v}) .
$$

Finally, in order to verify that our assumptions reduce to those of Theorem 2.2, we need to compute $\operatorname{Im} f^{\prime}(p)$. Let $\left(k_{1}, k_{2}, s\right)$ be an element of $\operatorname{Im} f^{\prime}(p) \subseteq \operatorname{Im} A \times Y_{2} \times \mathbb{R}$. It is easy to see that the vectors of the form $\left(k_{1}, 0,0\right)$ and $(0,0, s)$ belong to $\operatorname{Im} f^{\prime}(p)$. Thus, $\operatorname{Im} A \times\{0\} \times\{0\} \subseteq \operatorname{Im} f^{\prime}(p)$ and $\{0\} \times\{0\} \times \mathbb{R} \subseteq \operatorname{Im} f^{\prime}(p)$. Moreover, also the vector $\left(0, C_{2}(0, q), 0\right)$ belongs to the image of $f^{\prime}(p)$, so that $\operatorname{Im} f^{\prime}(p)$ contains the subspace $\operatorname{Im} A \times \operatorname{span}\left(C_{2}(0, q)\right) \times \mathbb{R}$, whose codimension in $\operatorname{Im} A \times Y_{2} \times \mathbb{R}$ is equal to $\operatorname{dim} T_{p} M$ (recall that $\operatorname{dim} Y_{2}=\operatorname{dim} \operatorname{Ker} A=\operatorname{dim} T_{p} M+1$ ). On the other hand, since $f^{\prime}(p)$ is Fredholm of index 1, by (3.7) we get codim $\operatorname{Im} f^{\prime}(p)=\operatorname{dim} T_{p} M$ as well. Therefore, the 
equality

$$
\operatorname{Im} f^{\prime}(p)=\operatorname{Im} A \times \operatorname{span}\left(C_{2}(0, q)\right) \times \mathbb{R}
$$

holds. Now, by interpreting the assumption

$$
A \dot{h}=0, \quad g^{\prime}(\bar{x}) \dot{h}=0 \quad \text { and } \quad B^{\prime}(\bar{x}) \dot{h}-\bar{\mu} C \dot{h} \in \operatorname{span}\{\operatorname{Im} A, C \bar{x}\} \Longrightarrow \dot{h}=0
$$

with the notation introduced here, we get

$$
\begin{aligned}
& \partial_{2} B_{2}(0, q) \dot{x}_{2}-\bar{\mu} C_{2}\left(0, \dot{x}_{2}\right)=\alpha C_{2}(0, q) \text { for some } \alpha \in \mathbb{R}, \\
& \text { and } \partial_{2} g(0, q) \dot{x}_{2}=0 \Longrightarrow \dot{x}_{2}=0 .
\end{aligned}
$$

In other words, if $\dot{h} \in T_{\bar{x}} S_{A}$ and $\varphi^{\prime}(0, q) \dot{h}=f^{\prime \prime}(0,0,0, q)(\dot{u},(0,0, \dot{h}))$ belongs to $\operatorname{Im} f^{\prime}(0,0,0, q)=\operatorname{Im} A \times \operatorname{span}\left(C_{2}(0, q)\right) \times \mathbb{R}$, then $\dot{h}=0$, that is the sufficient condition stated in Theorem 2.2 is satisfied. Consequently, $p=(0,0,0, q)$ is a bifurcation point (from $M$ ) of $f\left(\varepsilon, \lambda, x_{1}, x_{2}\right)=(0,0)$ or, equivalently, $\bar{x}=(0, q)$ is a bifurcation point (from $S_{A}$ ) of (3.1) as claimed.

An equivalent formulation of the assumption of Theorem 3.4 above is stated in the following remark.

REMARK 3.5. Let $Y_{\bar{x}}$ be the $m$-dimensional quotient space $Y / \operatorname{span}\{\operatorname{Im} A, C \bar{x}\}$ and denote $\pi_{\bar{x}}: Y \rightarrow Y_{\bar{x}}$ the canonical projection. Then $\bar{x}$ is a bifurcation point (from $S_{A}$ ) of (3.1), provided that

$$
A \dot{h}=0, \quad g^{\prime}(\bar{x}) \dot{h}=0 \quad \text { and } \quad \pi_{\bar{x}}\left(B^{\prime}(\bar{x})-\bar{\mu} C \dot{h}\right)=0 \Longrightarrow \dot{h}=0 .
$$

REMARK 3.6. From the proof of Theorem 2.2 given in [3], one can also deduce that the closure of the set of nontrivial solutions of (3.1) is, in fact, a $C^{1}$ curve in a neighborhood of the bifurcation point $(0,0, \bar{x})$. Let us show that this curve can be parametrized by $\varepsilon$, the first component of the space $\mathbb{R} \times \mathbb{R} \times X$. More precisely, let us decompose the Banach space $\mathbb{R} \times \mathbb{R} \times X$ into the one dimensional subspace $\mathbb{R} \times\{0\} \times\{0\}$ and its direct summand $\{0\} \times \mathbb{R} \times X$. We claim that the given curve can be represented by a parametrization having $\mathbb{R} \times\{0\} \times\{0\}$ as domain and $\{0\} \times \mathbb{R} \times X$ as codomain. It is a known fact that this can be done provided that the tangent vector to the curve at $(0,0, \bar{x})$ does not belong to $\{0\} \times \mathbb{R} \times X$. Such a vector belongs to $\operatorname{Ker} f^{\prime}(0,0, \bar{x})$ but it is not tangent to $M$. Thus, as already proved, its first component is nonzero, as required.

Remark 3.7. If $X=Y$ and $A$ is such that $\operatorname{Ker} A \oplus \operatorname{Im} A=X$ (this is the case when, for instance, $X$ is Hilbert and $A$ is selfadjoint with $\operatorname{Im} A$ closed), then one can identify $\operatorname{Ker} A$ with $\operatorname{coKer} A$ and the finite dimensional reduction acts between the same spaces. If, in addition, $C$ is the identity and $B$ is linear, then the necessary condition states that $\bar{x}$ is an eigenvector of $\left.B\right|_{\operatorname{Ker} A}: \operatorname{Ker} A \rightarrow \operatorname{Ker} A$ corresponding to some (real) eigenvalue $\bar{\mu}$. One can check that the sufficient condition stated in Theorem 3.4 means that the eigenvalue $\bar{\mu}$ is simple.

As will be clear in the next section, the special case when the spaces $X$ and $Y$ are finite dimensional and the operator $A$ is trivial is of some interest. In this situation our 
problem becomes

$$
\left\{\begin{array}{l}
\varepsilon B(x)=\lambda C x, \\
g(x)=0,
\end{array}\right.
$$

and Theorems 3.2 and 3.4 take the following form.

Corollary 3.8. Let $\operatorname{dim} X=\operatorname{dim} Y<\infty$. Assume $B$ of class $C^{1}$ on an open neighborhood of $g^{-1}(0)$ and $C$ invertible. Then, a necessary condition for $\bar{x} \in g^{-1}(0)$ to be a bifurcation point of (3.11) (from $g^{-1}(0)$ ) is that there exists $\bar{\mu} \in \mathbb{R}$ such that

$$
B(\bar{x})=\bar{\mu} C \bar{x} .
$$

Corollary 3.9. Let $\operatorname{dim} X=\operatorname{dim} Y<\infty$. Assume $B$ and $g$ of class $C^{2}$ on an open neighborhood of $g^{-1}(0)$ and $C$ invertible. Let $\bar{x} \in g^{-1}(0)$ and $\bar{\mu} \in \mathbb{R}$ be such that $B(\bar{x})=\bar{\mu} C \bar{x}$. Then, a sufficient condition for $\bar{x}$ to be a bifurcation point $\left(\right.$ from $\left.g^{-1}(0)\right)$ of (3.11) is that

$$
g^{\prime}(\bar{x}) \dot{h}=0 \quad \text { and } \quad B^{\prime}(\bar{x}) \dot{h}-\bar{\mu} C \dot{h} \in \operatorname{span}(C \bar{x}) \Longrightarrow \dot{h}=0 .
$$

4. Main results reformulated. This section is devoted to present practical reformulations of the two conditions for bifurcation given by Theorems 3.2 and 3.4. This involves a choice of linear coordinates for the spaces $\operatorname{Ker} A$ and $\operatorname{coKer} A$.

Recall that $\operatorname{Ker} A$ is nontrivial and let $m+1$ denote its dimension. A system of linear coordinates for $\operatorname{Ker} A$ is just a linear operator $J: \mathbb{R}^{m+1} \rightarrow X$ such that $\operatorname{Im} J=$ $\operatorname{Ker} A$. Since $\operatorname{dim} \operatorname{Ker} A=m+1$, this identifies $\operatorname{Ker} A$ with $\mathbb{R}^{m+1}$ by means of a linear isomorphism.

As regards coKer $A$, a system of linear coordinates may be given by considering a bounded linear operator $Q: Y \rightarrow \mathbb{R}^{m+1}$ such that $\operatorname{Ker} Q=\operatorname{Im} A$. In fact, in this case, there exists a unique linear operator $\hat{Q}: \operatorname{coKer} A \rightarrow \mathbb{R}^{m+1}$ such that $\hat{Q} \pi=Q$, where $\pi: Y \rightarrow \operatorname{coKer} A$ is the canonical projection. This operator is clearly injective and, consequently, must be surjective since $\operatorname{dim} \operatorname{coKer} A=m+1$ (recall that $A$ is Fredholm of index zero). In other words, $Q$ induces on $\operatorname{coKer} A$ a system of linear coordinates $\hat{Q}$. By abuse of terminology, we will say that $Q$ is a system of linear coordinates for $\operatorname{coKer} A$.

Theorem 4.1 below is a practical reformulation of the necessary condition given in Theorem 3.2.

THEOREM 4.1. Let, respectively, $J: \mathbb{R}^{m+1} \rightarrow X$ and $Q: Y \rightarrow \mathbb{R}^{m+1}$ be two systems of linear coordinates for $\operatorname{Ker} A$ and $\operatorname{coKer} A$, and define

$$
\bar{g}: \mathbb{R}^{m+1} \rightarrow \mathbb{R}, \quad \bar{B}: \mathbb{R}^{m+1} \rightarrow \mathbb{R}^{m+1}, \quad \bar{C}: \mathbb{R}^{m+1} \rightarrow \mathbb{R}^{m+1}
$$

by $\bar{g}=g J, \bar{B}=Q B J, \bar{C}=Q C J$.

If $\bar{a} \in \bar{g}^{-1}(0)$ is such that $J \bar{a}$ is a bifurcation point of (3.1), then there exists $\bar{\mu} \in \mathbb{R}$ such that

$$
\bar{B}(\bar{a})=\bar{\mu} \bar{C} \bar{a} .
$$

Proof. Since $\operatorname{Ker} Q=\operatorname{Im} A$, there exists a unique linear operator $\hat{Q}: \operatorname{coKer} A \rightarrow$ $\mathbb{R}^{m+1}$ such that $\hat{Q} \pi=Q$, where $\pi: Y \rightarrow \operatorname{coKer} A$ is the canonical projection. By 
Remark 3.3 with $\bar{x}=J \bar{a}$, there exists $\bar{\mu} \in \mathbb{R}$ such that $\pi B(J \bar{a})=\bar{\mu} \pi C J \bar{a}$. Thus, by applying $\hat{Q}$, one has $\hat{Q} \pi B(J \bar{a})=\bar{\mu} \hat{Q} \pi C J \bar{a}$, i.e. $\bar{B}(\bar{a})=\bar{\mu} \bar{C} \bar{a}$.

Remark 4.2 below will come on hand when one seeks for a system of linear coordinates for $\operatorname{coKer} A$.

REMARK 4.2. If we assume that a bounded linear operator $Q: F \rightarrow \mathbb{R}^{m+1}$ is onto, then clearly codim $\operatorname{Ker} Q=m+1$, the same as $\operatorname{Im} A$. Thus, the following three conditions are equivalent:

- $\operatorname{Ker} Q=\operatorname{Im} A$;

- $\operatorname{Ker} Q \subseteq \operatorname{Im} A$;

- $\operatorname{Ker} Q \supseteq \operatorname{Im} A$.

In the same spirit of Theorem 4.1, let us now state the "linear coordinates" version of the sufficient condition given in Theorem 3.4.

TheOREM 4.3. Let $J, Q, \bar{B}, \bar{C}$ and $\bar{g}$ be as in Theorem 4.1. Let $\bar{a} \in \bar{g}^{-1}(0)$ and $\bar{\mu} \in \mathbb{R}$ be such that $\bar{B}(\bar{a})=\bar{\mu} \bar{C} \bar{a}$. Then Ja is a bifurcation point of (3.1), provided that

$$
\dot{a} \in \mathbb{R}^{m+1}, \quad \bar{g}^{\prime}(\bar{a}) \dot{a}=0 \quad \text { and } \quad \bar{B}^{\prime}(\bar{a}) \dot{a}-\bar{\mu} \bar{C} \dot{a} \in \operatorname{span}(\bar{C} \bar{a}) \Longrightarrow \dot{a}=0 .
$$

Proof. The assertion follows immediately noting that $\bar{x}=J \bar{a}$ and $\dot{h}=J \dot{a}$ clearly satisfy the sufficient condition of Theorem 3.4.

The following easy consequence of Theorem 4.3 could be useful in applications as shown, for instance, in the next section.

Corollary 4.4. Let $J, Q, \bar{B}, \bar{C}$, $\bar{g}$ be as in Theorem 4.1, $\bar{a} \in \bar{g}^{-1}(0)$, and $\bar{\mu} \in \mathbb{R}$ be such that $\bar{B}(\bar{a})=\bar{\mu} \bar{C} \bar{a}$. Assume that $R: \mathbb{R}^{m+1} \rightarrow \mathbb{R}^{m}$ is a surjective linear operator such that $R \bar{C} \bar{a}=0$. Then Ja is a bifurcation point of (3.1), provided that

$$
\dot{a} \in \mathbb{R}^{m+1}, \quad \bar{g}^{\prime}(\bar{a}) \dot{a}=0 \quad \text { and } \quad R\left(\bar{B}^{\prime}(\bar{a})-\bar{\mu} \bar{C}\right) \dot{a}=0 \Longrightarrow \dot{a}=0 .
$$

Proof. It is enough to show that $\operatorname{Ker} R=\operatorname{span}(\bar{C} \bar{a})$. By assumption, $\bar{C} \bar{a}$ belongs to $\operatorname{Ker} R$, which is 1 -dimensional since $R$ is onto. Thus, $\operatorname{Ker} R$ must coincide with $\operatorname{span}(\bar{C} \bar{a})$, as claimed.

Remark 4.5. Consider the map $a \in \bar{g}^{-1}(0) \mapsto R(\bar{B}(a)-\bar{\mu} \bar{C} a) \in \mathbb{R}^{m}$. Clearly, the necessary condition of Theorem 4.1 implies that $\bar{a}$ is a zero of this map. The sufficient condition (4.2) of Corollary 4.4 above, means that such a zero is nondegenerate.

REMARK 4.6. It should be observed that Theorems 4.1 and 4.3 above contain a reduction of the general case to a problem in $\mathbb{R}^{m+1}$ analogous to the finite dimensional problem presented in (3.11). In fact, it is easy to see that the necessary and the sufficient conditions obtained in these theorems are the same as those proved in Corollaries 3.8 and 3.9.

5. An example. In this section, we give an application of the previous bifurcation results to the second order differential equation, depending on the real parameters $\varepsilon$ and $\lambda$,

$$
x^{\prime \prime}+x+\varepsilon\left(t x+x^{2}\right)=\lambda x, \quad t \in \mathbb{R} .
$$


We are interested in $2 \pi$-periodic solutions $x$ of (5.1) normalized as follows:

$$
\frac{1}{\pi} \int_{-\pi}^{\pi} x^{2}(t) d t=1
$$

In particular, we look for solutions which bifurcate from a given trivial (i.e. obtained for $\varepsilon=\lambda=0$ ) normalized solution; that is, from a solution of the type $x(t)=c \sin t+$ $d \cos t$, with $c^{2}+d^{2}=1$.

To this end, consider the problem

$$
\left\{\begin{array}{l}
x^{\prime \prime}+x+\varepsilon\left(t x+x^{2}\right)=\lambda x \\
x(-\pi)=x(\pi), \quad x^{\prime}(-\pi)=x^{\prime}(\pi) \\
\frac{1}{\pi} \int_{-\pi}^{\pi} x^{2}(t) d t=1
\end{array}\right.
$$

Clearly, all solutions of (5.2) are $C^{\infty}$ and we will look for them in the Banach space

$$
C_{2 \pi}^{2}=\left\{x \in C^{2}([-\pi, \pi]): x(-\pi)=x(\pi), x^{\prime}(-\pi)=x^{\prime}(\pi)\right\} .
$$

In the notation of Section 3 , set

$$
X=C_{2 \pi}^{2}, \quad Y=C([-\pi, \pi])
$$

and define

$$
\begin{gathered}
A: X \rightarrow Y \text { by } A x=x^{\prime \prime}+x, \\
B: X \rightarrow Y \text { by } B(x)(t)=t x(t)+x^{2}(t), \\
C: X \rightarrow Y \text { to be the inclusion, } \\
g: X \rightarrow \mathbb{R} \text { by } g(x)=\frac{1}{\pi} \int_{-\pi}^{\pi} x^{2}(t) d t-1 .
\end{gathered}
$$

As observed in the preliminaries, the operator $A$, which is clearly Fredholm of index 2 between $C^{2}([-\pi, \pi])$ and $Y$, becomes of index 0 , when restricted to the 2-codimensional closed subspace $X$ of $C^{2}([-\pi, \pi])$, with 2-dimensional kernel

$$
\operatorname{Ker} A=\{x \in X: x(t)=\alpha \sin t+\beta \cos t, \alpha, \beta \in \mathbb{R}\}
$$

and 2-codimensional image

$$
\operatorname{Im} A=\left\{y \in Y: \int_{-\pi}^{\pi} y(t) \sin t d t=0, \int_{-\pi}^{\pi} y(t) \cos t d t=0\right\} .
$$

Moreover, the operator $A-\lambda C$ given by $x \mapsto x^{\prime \prime}+(1-\lambda) x$ is clearly invertible for $\lambda \neq 0$ sufficiently small.

The first derivative of the operator $B$ at a point $x \in X$ along a vector $h \in X$ is given by

$$
\left(B^{\prime}(x) h\right)(t)=2 x(t) h(t)+t h(t) .
$$

Thus, the second derivative of $B$ at $x \in X$ along a pair of vectors $(h, k) \in X^{2}$ is represented by

$$
\left(B^{\prime \prime}(x)(h, k)\right)(t)=2 h(t) k(t)
$$


Hence $B^{\prime \prime}$ is a constant map from $X$ into the space $L_{2}(X, Y)$ of continuous bilinear operators from $X$ into $Y$, therefore $B$ is $C^{2}$, as required.

The real valued map $g$ is clearly $C^{\infty}$ and $g^{\prime}(x) x=\frac{2}{\pi} \int_{-\pi}^{\pi} x^{2}(t) d t \neq 0$ for any $x \in$ $g^{-1}(0)$. Moreover, as easily verified, for any $x \in X, x \neq 0$, there exists (a unique) $s>0$ such that $\frac{s^{2}}{\pi} \int_{-\pi}^{\pi} x^{2}(t) d t=1$, i.e. $g^{-1}(0)$ surrounds the origin.

Moreover, the manifold $S_{A}=\operatorname{Ker} A \cap g^{-1}(0)$ is given by

$$
S_{A}=\left\{x \in X: x(t)=\alpha \sin t+\beta \cos t, \alpha^{2}+\beta^{2}=1\right\}
$$

and is clearly 1-dimensional.

First, our aim is to apply Theorem 4.1 to system (5.2) in order to get a necessary condition for bifurcation. To this end, let us define $J: \mathbb{R}^{2} \rightarrow X$ as

$$
J(\alpha, \beta)=\alpha \sin t+\beta \cos t
$$

and $Q: Y \rightarrow \mathbb{R}^{2}$ as

$$
Q y=\frac{1}{\pi}\left(\int_{-\pi}^{\pi} y(t) \sin t d t, \int_{-\pi}^{\pi} y(t) \cos t d t\right) .
$$

Clearly, $\operatorname{Im} J=\operatorname{Ker} A$ and, as observed before, $y \in \operatorname{Im} A$ if and only if $Q y=0$, i.e. $\operatorname{Ker} Q=\operatorname{Im} A$. An easy computation shows that the map $\bar{g}=J g: \mathbb{R}^{2} \rightarrow \mathbb{R}^{2}$ is given by

$$
\bar{g}(\alpha, \beta)=\alpha^{2}+\beta^{2}-1
$$

and that the composition $\bar{C}=Q C J: \mathbb{R}^{2} \rightarrow \mathbb{R}^{2}$,

$$
\bar{C}(\alpha, \beta)=\frac{1}{\pi}\left(\int_{-\pi}^{\pi}(\alpha \sin t+\beta \cos t) \sin t d t, \int_{-\pi}^{\pi}(\alpha \sin t+\beta \cos t) \cos t d t\right),
$$

turns out to be the identity. Moreover,

$$
\bar{g}^{-1}(0)=J^{-1}\left(S_{A}\right)=\left\{(\alpha, \beta) \in \mathbb{R}^{2}: \alpha^{2}+\beta^{2}=1\right\} .
$$

Now, according to Theorem 4.1, a necessary condition for a solution

$$
\bar{x}(t)=\bar{\alpha} \sin t+\bar{\beta} \cos t,
$$

with $\bar{\alpha}^{2}+\bar{\beta}^{2}=1$, of (5.2) to be a bifurcation point from $S_{A}$ is that there exists $\bar{\mu} \in \mathbb{R}$ such that the pair $(\bar{\alpha}, \bar{\beta})$ of the unit circle $\bar{g}^{-1}(0)$ satisfies the eigenvalue problem

$$
\bar{B}(\alpha, \beta)=\bar{\mu} \bar{C}(\alpha, \beta),
$$

where $\bar{B}: \mathbb{R}^{2} \rightarrow \mathbb{R}^{2}$ is the composition $\bar{B}=Q B J$. By computing $\bar{B}$, one easily obtains

$$
\bar{B}(\alpha, \beta)=-\frac{1}{2}(\beta, \alpha) .
$$

Therefore, we are reduced to find the eigenvalues of the equation

$$
-\frac{1}{2}(\beta, \alpha)=\mu(\alpha, \beta)
$$


and the corresponding normalized eigenvectors $(\alpha, \beta)$, i.e. satisfying $\alpha^{2}+\beta^{2}=1$. By solving (5.3) we get the two values $\bar{\mu}_{-}=-\frac{1}{2}$, with corresponding pairs $\pm\left(\frac{\sqrt{2}}{2}, \frac{\sqrt{2}}{2}\right)$, and $\bar{\mu}_{+}=\frac{1}{2}$, with pairs $\pm\left(\frac{\sqrt{2}}{2},-\frac{\sqrt{2}}{2}\right)$.

Let us now show that such eigenvectors also satisfy the sufficient condition for bifurcation stated in Corollary 4.4. To this end, choose one of the previous eigenvalues, say, for instance, $\bar{\mu}_{-}$and define $R: \mathbb{R}^{2} \rightarrow \mathbb{R}$ by $R(\alpha, \beta)=\alpha-\beta$. Since the map $\bar{B}+$ $\frac{1}{2} \bar{C}: \mathbb{R}^{2} \rightarrow \mathbb{R}^{2}$ is given by

$$
\left(\bar{B}+\frac{1}{2} \bar{C}\right)(\alpha, \beta)=-\frac{1}{2}(\beta, \alpha)+\frac{1}{2}(\alpha, \beta)=\frac{1}{2}(\alpha-\beta, \beta-\alpha),
$$

by composing with $R$, we obtain the linear operator

$$
R\left(\bar{B}+\frac{1}{2} \bar{C}\right)(\alpha, \beta)=\alpha-\beta .
$$

Hence, its derivative at each one of the eigenvectors $\pm\left(\frac{\sqrt{2}}{2}, \frac{\sqrt{2}}{2}\right)$ along any vector $(\dot{\alpha}, \dot{\beta}) \in \mathbb{R}^{2}$ turns out to be

$$
R\left(\bar{B}^{\prime}+\frac{1}{2} \bar{C}\right)\left( \pm\left(\frac{\sqrt{2}}{2}, \frac{\sqrt{2}}{2}\right)\right)(\dot{\alpha}, \dot{\beta})=\dot{\alpha}-\dot{\beta} .
$$

In order to check condition (4.2) of Corollary 4.4, we need to restrict

$$
R\left(\bar{B}^{\prime}+\frac{1}{2} \bar{C}\right)\left( \pm\left(\frac{\sqrt{2}}{2}, \frac{\sqrt{2}}{2}\right)\right)
$$

to the 1-dimensional space

$$
\left\{(\dot{\alpha}, \dot{\beta}) \in \mathbb{R}^{2}: \bar{g}^{\prime}\left( \pm\left(\frac{\sqrt{2}}{2}, \frac{\sqrt{2}}{2}\right)\right)(\dot{\alpha}, \dot{\beta})=0\right\} .
$$

As easily seen by computing the derivative of $\bar{g}$ at $\pm\left(\frac{\sqrt{2}}{2}, \frac{\sqrt{2}}{2}\right)$, this space is given by

$$
\left\{(\dot{\alpha}, \dot{\beta}) \in \mathbb{R}^{2}: \dot{\alpha}+\dot{\beta}=0\right\} .
$$

Consequently, we are reduced to solve the system

$$
\left\{\begin{array}{l}
\dot{\alpha}-\dot{\beta}=0 \\
\dot{\alpha}+\dot{\beta}=0
\end{array}\right.
$$

that gives $\dot{\alpha}=\dot{\beta}=0$. This shows that (4.2) is satisfied in the case of the eigenvalue $\bar{\mu}_{-}$. A similar argument holds for $\bar{\mu}_{+}$.

\section{REFERENCES}

1. R. Chiappinelli, Isolated Connected Eigenvalues in Nonlinear Spectral Theory, Nonlinear Funct. Anal. Appl. 8 (2003), 557-579.

2. M. G. Crandall and P. H. Rabinowitz, Bifurcation from Simple Eigenvalues, J. Funct. Anal. 8 (1971), 321-340.

3. M. Furi, M. Martelli and M. P. Pera, General Bifurcation Theory: Some Local Results and Applications, Differential Equations and Applications to Biology and to Industry (Cooke K., Cumberbatch E., Martelli M., Tang B. and Thieme H. Editors), (World Scientific, 1996), $101-115$. 
4. M. Furi and M. P. Pera, Co-Bifurcating Branches of Solutions for Nonlinear Eigenvalue Problems in Banach Spaces, Ann. Mat. Pura Appl. 135 (1983), 119-131.

5. M. Furi and M. P. Pera, Bifurcation of Fixed Points from a Manifold of Trivial Fixed Points, Nonlinear Funct. Anal. Appl. 11 (2006), 265-292. 1976)

6. M. W. Hirsch, Differential Topology, Graduate Texts in Math. 33, (Springer-Verlag,

7. S. Lang, Introduction to Differentiable Manifolds, (Interscience Publishers, John Wiley \& Sons, Inc., New York, 1966).

8. M. Martelli, Large oscillations of forced nonlinear differential equations, AMS Cont. Mathematics 21 (1983), 151-159.

9. L. Nirenberg, Topics in Nonlinear Functional Analysis, Courant Institute of Mathematical Sciences, (AMS, New York, 2001). 